\title{
Comportamento de Cilindros de Carbono/Epóxi Submetidos a Cargas Compressivas Axiais
}

\author{
Adriano Gonçalves \\ Centro Técnico Aeroespacial CTAIAE/GER-V
}

Sérgio F. M. Almeida

Centro Técrico Aeroespacial CTAITAIEM

\section{Flaminio Levy Neto Universidade de Brasilia UnB/FT/ENM}

Resumo: Para estruturas utilizadas no setor aeroespacial, os requisitos de baixo peso, alta resistência e rigidez, além de estabilidade dimensional, têm propiciado o aumento da utilização de materiais compósitos nas suas manufaturas. Em particular, cascas cilíndricas ou estruturas construídas pela junção de cilindros de paredes finas, confeccionadas em fibra de carbono e resina epóxi, são amplamente utilizadas neste tipo de aplicação. Neste trabalho, um programa experimental foi desenvolvido para determinar as tensões de falha, os módulos de elasticidade e o modo de falha de 47 cilindros com diâmetro interno de $40 \mathrm{~mm}$ e espessura nominal de 0,6 $\mathrm{mm}$ (com exceção de 2 corpos de prova), fabricados em carbono/epóxi, quando submetidos a cargas compressivas uniaxiais. Os espécimes testados possuíam diferentes razões entre comprimento e diâmetro (variando de 2,50 a 11,25) e seqüências de laminação variadas (orientações de camadas). Os resultados dos ensaios foram comparados aos obtidos em análises realizadas com programas de elementos finitos e os fatores que influenciaram o comportamento mecânico destes cilindros foram analisados.

Palamraschave Material compósito, compressão, fibra de carbono, epóxi, cilindro.

\section{Mechanical Behavior of Carbon/Epoxy Cylinders Under Axial Compressive Loads}

Abstract: The requirements of low weight and dimensional stability, combined with high strength and stiffness, for aerospace structures has prompted an increasing use of fiber reinforced materials in manufacturing such structures. In particular, carbon/epoxy cylinders have been widely used in aerospace applications. In this work, an experimental program was developed to determine failure loads, modulus of elasticity and failure modes of 47 carbon/epoxy cylinders shells under compressive loads. The specimens tested had several different length/diameter (from 2.50 to 11.25) ratios and laminate lay-up. These results were compared to the analytical results from finite element code and the most important factors influencing the mechanical behavior of this type of structure were analyzed.

Keyuords: Composite materials, compression, carbon fiber, epoxy, cylinder.

\section{Introdução}

Em virtude de possuírem elevados índices de resistência mecânica e rigidez por unidade de peso, além de não sofrerem corrosão galvânica em muitas situações práticas, laminados de matriz plástica reforçada com fibras vem sendo crescentemente empregados na fabricação de tubulações e vasos de pressão, dentre outros componentes estruturais axissimétricos ${ }^{[1]}$. No caso de cascas axissimétricas de carbono/epóxi submetidas a cargas compressivas, vários são os possíveis mecanismos de falha, podendo-se citar: (i) flambagem

Autor para correspondência: Sérgio F. M. de Almeira, Centro Técnico Aeroespacial, ITA, IEM, CEP: 12228-900, São José dos Campos, SP. E-mail: frascino@mec.ita.cta.br. 
com bifurcação, com aparecimento de ondas circunferenciais; (ii) colapso axissimétrico ${ }^{[2]}$; (iii) falha do material na qual a ruptura da primeira camada (first ply failure, FPF) determina a ruptura final e definitiva do laminado; e (iv) falha do material com resistência residual após FPF, implicando na ruptura de sucessivas camadas do laminado (last ply failure, LPF), antes do componente perder por completo sua resistência mecânica $^{[3,4]}$. Quando a casca é cilíndrica, a predominância de um dentre os possíveis mecanismos de falha depende de fatores tais como, por exemplo, relação Diâmetro/espessura $(D / t)$ do cilindro, orientações das fibras, bem como das frações volumétricas e propriedades dos constituintes do laminado.

As falhas que ocorrem quando cilindros de parede fina (relação $D / t$ elevada) de materiais compósitos confeccionados em fibra de carbono e resina epóxi são submetidos a cargas compressivas axiais, são, na maioria das vezes, resultado de instabilidades locais, que podem ser observadas tanto em escala macroscópica (fratura, flambagem local) quanto em escala microscópica (microflambagem de fibras). Além dos citados anteriormente, vários outros trabalhos teóricos e experimentais foram realizados descrevendo alguns mecanismos das falhas presentes neste tipo de estrutura e sob esta solicitação ${ }^{[5-7]}$. Observa-se, no entanto, que o modo de falha mais presente, está associado a instabilidades macroscópicas, envolvendo tanto o cilindro como um todo, bem como somente em partes específicas do mesmo.

Constata-se que para cilindros sujeitos a compressão, há discrepâncias entre os valores obtidos em análises teóricas e os obtidos em ensaios, podendo estas serem bastante elevadas e ocorrerem em termos do valor da carga de ruptura ou flambagem e nos valores das deformações longitudinais e transversais ${ }^{[5]}$. Em casos estudados, verificou-se que as cargas obtidas experimentalmente apresentavam valores inferiores a $50 \%$ das calculadas teoricamente ${ }^{[5]}$. Esta discrepância está associada à extrema sensibilidade das estruturas com flambagem por bifurcação à existência de imperfeições e desvios geométricos decorrentes de seu processo de fabricação. Imperfeições geométricas da ordem de $20 \%$ da espessura da parede podem causar uma redução de mais de $50 \%$ na carga de flambagem de cilindros de parede fina ${ }^{[8]}$. Portanto, em aplicações práticas, é essencial caracterizar experimentalmente o efeito das imperfeições associadas ao processo de fabricação empregado.
Os modos de falha observados quando cilindros de carbono/epóxi são submetidos, especificamente, a um carregamento axial, podem enquadrar-se nos seguintes casos:

- a fratura do laminado ocorre, predominantemente, num plano perpendicular ao carregamento;

- há uma presença significativa de trincas as quais se propagam pela matriz (resina), paralelamente à direção das fibras;

- há flambagem local do laminado, seja em parte ou totalmente ao redor da circunferência do cilindro; e

- há flambagem de coluna (ou Flambagem de Euler).

O presente trabalho visa investigar, experimentalmente, o comportamento mecânico (rigidez, resistência e modos de falha) de cilindros bi-engastados submetidos a cargas compressivas uniaxiais, confeccionados em resina epóxi reforçada por fibra de carbono. Foram testados cilindros com diversos comprimentos e orientação do reforço de suas camadas. Simulações numéricas, utilizando programas de elementos finitos, foram realizadas para se poder correlacionar os resultados obtidos teórica e experimentalmente.

\section{Materiais e Métodos}

Foram fabricados e ensaiados em compressão axial quarenta e sete cilindros de diâmetro $D=40 \mathrm{~mm}$, confeccionados com fitas de fibra de carbono unidirecionais, pré-impregnadas com resina epóxi. Os cilindros foram agrupados nos tipos I, II, III e IV em função do comprimento e da orientação das fibras relativamente às direções meridionais dos mesmos, conforme indicado na Tabela 1 . Utilizou-se $L$ para designar-se o comprimento e $D$ para os diâmetros dos cilindros.

Os ensaios visaram obter experimentalmente, até a ruptura, as tensões, deformações e o modo de falha dos cilindros, cujas configurações estão indicadas na Tabela 1.

As fitas utilizadas na produção dos cilindros são de fibras de carbono unidirecionais pré-impregnadas com resina epóxi, produzidas pela Hexcel Advanced Composites (código CMS 91010 tipo 1), tendo espessura nominal de $0,09 \mathrm{~mm}$. A utilização de fitas préimpregnadas (tapes) confere ao laminado uma 
Tabela 1. Características dos corpos de prova.

\begin{tabular}{|c|c|c|c|c|c|}
\hline $\begin{array}{c}\text { Orientação das } \\
\text { camadas }(*) \\
(D / t)\end{array}$ & Identificação & $\begin{array}{c}D \text { interno } \\
(\mathrm{mm})\end{array}$ & $\begin{array}{c}D \text { externo } \\
(\mathbf{m m})\end{array}$ & $\begin{array}{c}L \\
(\mathrm{~mm})\end{array}$ & Quantidade \\
\hline \multirow[t]{2}{*}[0_{6}]{} & $\mathrm{I}-1$ & 40 & 41,2 & 100 & 05 \\
\hline & $\mathrm{I}-2$ & 40 & 41,2 & 200 & 05 \\
\hline (67) & $\mathrm{I}-3$ & 40 & 41,2 & 300 & 05 \\
\hline \multirow[t]{2}{*}[0/\pm45]{$\mathrm{s}$} & II-1 & 40 & 41,2 & 100 & 05 \\
\hline & II-2 & 40 & 41,2 & 200 & 05 \\
\hline (67) & II-3 & 40 & 41,2 & 300 & 05 \\
\hline \multirow[t]{2}{*}[0/\pm60]{$\mathrm{s}$} & IIII-1 & 40 & 41,2 & 100 & 05 \\
\hline & III-2 & 40 & 41,2 & 200 & 05 \\
\hline (67) & III-3 & 40 & 41,2 & 300 & 05 \\
\hline$[ \pm 45] \mathrm{T}$ & & & & & \\
\hline (200) & IV-4 & 40 & 40,4 & 450 & 02 \\
\hline
\end{tabular}

(*) Refere-se ao ângulo formado entre as fibras e o direção longitudinal (meridiano) do cilindro

distribuição adequada e uniforme de fibras e resina, garantindo uma espessura constante e consequentemente uma estrutura com qualidade elevada.

A orientação das camadas é fundamental no sentido de conseguir-se um alinhamento adequado das fibras para que se possa representar fielmente os ângulos que foram utilizados nos cálculos teóricos. Desta forma, especial atenção foi tomada no sentido de garantir que as peças em questão apresentassem suas fibras orientadas convenientemente, pois qualquer variação angular apresentaria influência direta nos resultados dos ensaios devido à alta anisotropia do material.

Além da orientação das camadas, a espessura e o diâmetro dos cilindros tem influência direta nos valores das tensões, deformações e no modo de falha. Foi realizada uma inspeção dimensional de cada espécime. O comprimento e o diâmetro externo dos cilindros tiveram um desvio máximo de $0,1 \%$ e $0,4 \%$ em relação ao valor nominal, respectivamente ${ }^{[9]}$. A utilização de pré-impregnados e um mandril com bom acabamento na produção dos cilindros levaram a um bom controle dimensional e bom acabamento dos espécimes.

Como indicado na Tabela 1, os cilindros possuíam seis camadas (exceto os do tipo IV, que possuíam apenas duas camadas) de fitas pré-impregnadas, que foram laminadas manualmente uma a uma, respeitando a orientação desejada, sobre um ferramental apropriado, fabricado em liga de alumínio 6061, denominado mandril. Este ferramental foi projetado visando permitir a obtenção de cilindros sem variação de diâmetro interno e que também possibilitasse a aplicação de vácuo durante todo o processo de polimerização da resina.

A qualidade dos cilindros e a obtenção das propriedades físicas e mecânicas esperadas para o laminado, dependem da correta execução do ciclo de polimerização da resina. $\mathrm{O}$ ciclo de cura foi realizado em duas etapas sendo, a primeira à temperatura de $80^{\circ} \mathrm{C}$ por 30 minutos, seguida de outra etapa a $120^{\circ} \mathrm{C}$ por 90 minutos. Após esta etapa seguia-se um resfriamento lento, minimizando-se, desta forma, a criação de tensões térmicas. Durante as etapas de aquecimento, as peças foram submetidas a vácuo (cerca de $10^{5} \mathrm{~Pa}$ ).

Após a retirada dos cilindros do mandril, os mesmos são usinados em torno horizontal, sendo que a única usinagem a ser efetuada diz respeito ao faceamento das extremidades dos tubos para conseguir-se os comprimentos desejados, pois, os diâmetros interno e externo são obtidos no processo de laminação. Pelo caráter abrasivo do material dos cilindros, e para evitar o aparecimento de delaminações em suas extremidades, utilizam-se pastilhas adiamantadas, que propiciam excelente acabamento superficial. Após a usinagem os cilindros são identificados, medidos os seus comprimentos, diâmetros externos, espessuras e suas massas.

Estudos indicam que materiais unidirecionais submetidos à compressão, podem falhar a um baixo nível de tensão ${ }^{[10]}$ por deterioração dos filamentos das extremidades (filament brooming). Para prevenir este efeito, foram desenvolvidos terminais de aço SAE 4340 com diâmetro de $80 \mathrm{~mm}$ e espessura de $20 \mathrm{~mm}$, onde foram encapsuladas as extremidades dos cilin- 
dros. Os encapsulamentos foram realizados por meio da deposição de resina epóxi de cura a frio, LY553/ HY951 (Ciba-Geigy), no interior dos terminais, estando os cilindros alinhados convenientemente para permitir, posteriormente durante o ensaio, a correta aplicação do carregamento. É importante ressaltar que a polimerização desta resina processa-se à temperatura ambiente ${ }^{[4]}$.

Em cada cilindro foram colados extensômetros elétricos (strain-gages) para determinação das deformações, sendo dois no centro do cilindro na direção longitudinal, defasados circunferencialmente de $180^{\circ}$, e um, também no centro, na direção circunferencial.

Os ensaios consistiram em comprimir os cilindros axialmente (i.e. na direção longitudinal) utilizando-se a máquina de ensaios marca Otto Wolpert, tipo THZ-200 (capacidade $200 \mathrm{kN}$ ). A carga foi aplicada com velocidade de $0,75 \mathrm{~mm} / \mathrm{min}$, através do deslocamento da base superior da máquina. Durante todo o ensaio foram registradas as deformações dos extensômetros em função da carga aplicada.

\section{Análise Teórica}

A análise teórica de cilíndricos compostos de multicamadas de fibras de carbono em matriz de epóxi, submetidos a cargas compressivas uniaxiais, foi realizada utilizando-se dois programas de elementos finitos. Para análise de resistência dos cilindros foi utilizado o programa COMPSHELL ${ }^{[4]}$. A análise de flambagem foi realizada pelo programa MSC/NASTRAN.

As propriedades mecânicas do material carbono/ epóxi foram obtidas ensaiando-se corpos de prova
Tabela 2. Propriedades mecânicas do material.

\begin{tabular}{lc}
\hline \multicolumn{1}{c}{ Propriedade } & Valor \\
\hline Módulo de elasticidade longitudinal, $E_{11}(\mathrm{GPa})$ & 170 \\
Módulo de elasticidade transversal, $E_{22}(\mathrm{GPa})$ & 12,4 \\
Módulo de cisalhamento no plano, $G_{12}(\mathrm{GPa})$ & 7,1 \\
Coeficiente de Poisson no plano, $V_{12}$ & 0,27 \\
Resistência longitudinal à compressão, $X_{1 C}(\mathrm{MPa})$ & 661 \\
Resistência transversal à tração, $X_{2 T}(\mathrm{MPa})$ & 16 \\
Resistência transversal à compressão, $X_{2 C}(\mathrm{MPa})$ & 126 \\
Resistência ao cisalhamento no plano, $S_{12}(\mathrm{MPa})$ & 40 \\
\hline
\end{tabular}

unidirecionais segundo as normas ASTM D-3039-76 e D-3410-87. Os resultados estão indicados na Tabela $2^{[9]}$.

O programa COMPSHELL baseia-se na teoria geometricamente não linear de cascas de Novozhilov (i.e. permite deformações moderadamente grandes ${ }^{[2]}$ ) onde admite-se que o material é ortotrópico, a casca é fina (o quociente $D / t$ é elevado), axissimétrica e composta de várias camadas. As camadas podem ser de materiais com propriedades diferentes e o eixo principal em cada camada (i.e. direção das fibras) pode ter qualquer orientação relativamente ao eixo longitudinal da casca. A espessura da parede é considerada muito menor que o raio de curvatura da superfície de referência das cascas, ou seja $(t<D / 2)$, consequentemente, o estado tridimensional de tensões pode ser reduzido a um estado bidimensional equivalente ${ }^{[2]}$.

O programa calcula as tensões e deformações ao longo da estrutura. As deformações planares em cada camada podem ser rotacionadas das direções circunferencial e meridional do cilindro $(x, y)$ para as dire-

Tabela 3. Características dos modelos de elementos finitos.

\begin{tabular}{ccccc}
\hline Identificação & $\mathbf{A}\left(\mathbf{m m}^{2}\right)$ & Autovalor & $\mathbf{N}^{\mathbf{o}}$ de Nós & $\mathbf{N}^{\mathbf{o}}$ de Elementos \\
\hline I-1 & 76,53 & 1,812 & 504 & 480 \\
I-2 & 76,53 & 1,781 & 984 & 960 \\
I-3 & 76,53 & 1,774 & 1464 & 1440 \\
III-1 & 76,53 & 3,073 & 504 & 480 \\
II-2 & 76,53 & 2,415 & 984 & 960 \\
II-3 & 76,53 & 1,911 & 1464 & 1440 \\
III-1 & 76,53 & 3,095 & 504 & 480 \\
III-2 & 76,53 & 2,590 & 984 & 960 \\
III-3 & 76,53 & 2,157 & 1464 & 1440 \\
IV-4 & 25,46 & 0,608 & 2184 & 2160 \\
\hline
\end{tabular}


ções principais das lâminas $(1,2)$. Usando-se as relações tensão-deformação para cada camada, as respectivas tensões podem ser obtidas e desta forma, um critério de falha pode ser então utilizado para avaliar a integridade do material em cada camada. Neste trabalho foram analisadas as falhas pelos critérios de Hoffman e da Deformação Máxima, considerando a falha total como a falha da primeira camada first ply failure (FPF) ${ }^{[3,4,7]}$.

O programa discretiza a estrutura em elementos (anéis axissimétricos de espessura uniforme). A estrutura deve ser composta de uma combinação de elementos tendo raio de curvatura meridional constante (esférico, cilíndrico, toroidal ou cônico). Estes elementos axissimétricos contêm dois nós nas extremidades, sendo que cada nó tem quatro graus de liberdade $(u, v, w$ e $\beta$, ou seja, translações meridional, circunferencial e radial, bem como rotação meridional, respectivamente) referidos à superfície média do elemento. As funções de interpolação são cúbicas para $w$, quadráticas para $\beta$ e lineares para $u \mathrm{e} v$. O algoritmo de Newton-Raphson é usado para resolver este sistema de equações não lineares ${ }^{[4,7]}$.

As cargas de flambagem linear foram obtidas utilizando-se modelos em elementos finitos gerados pelo pré-processador MSC/XL e analisados pelo programa MSC/NASTRAN. Esses modelos são capazes de prever: colapso axissimétrico, flambagem com bifurcação, e flambagem de coluna. Os modelos em elementos finitos dos cilindros contém somente elementos de placa CQUAD4. Os cilindros foram considerados engastados na base, sendo que no topo apenas o grau de liberdade, correspondente à translação no sentido longitudinal, foi liberado.

Para o cálculo das tensões de flambagem foi arbitrado o valor de $24000 \mathrm{~N}$ para a força inicial $\left(F_{i}\right)$, exceto para o caso IV-4 onde $F_{i}=4800 \mathrm{~N}$. O programa MSC/NASTRAN fornece como saída de resultados, o autovalor associado à carga de flambagem correspondente a cada configuração de cilindro. Os resultados dos autovalores bem como os números de nós, de elementos e as áreas transversais $(A)$ dos cilindros, estão mostrados na Tabela 3 . O problema de flambagem linearizado é colocado na forma de um problema de autovalor $^{[11]}$.

$$
\left[[K]-\lambda\left[K_{G}\right]\right]\{\phi\}=\{0\}
$$

onde $[K]$ é a matriz de rigidez global da estrutura e
$\left[K_{G}\right]$ é a matriz de rigidez geométrica global da estrutura que é proporcional à força inicial $F_{i}$, arbitrada conforme descrito acima. $\mathrm{O}$ autovetor $\{\phi\}$ define $\mathrm{o}$ modo de flambagem e o autovalor $\lambda$ está relacionado com a carga de flambagem, $F_{f l}$, por:

$$
F_{f l}=\lambda F_{i}
$$

O cálculo da tensão de flambagem, $\sigma_{f l}$, foi realizado utilizando-se a seguinte relação:

$$
\sigma_{f l}=F_{f l} / A
$$

onde $A$ é a área da seção transversal da casca cilíndrica.

\section{Resultados}

Foram verificados nos ensaios quatro modos de falha, sendo identificados como:

Ruptura das Fibras: Este modo de falha foi observado nos cilindros tipo II e III, indepentemente dos comprimentos dos mesmos. Observa-se que a fratura ocorre perpendicularmente ao eixo de aplicação da carga (Figura 1).

Flambagem Local Acoplada à Fratura da Matriz: Este modo de falha apresentou-se somente nos cilindros do tipo I, em todos eles as tensões de falha experimentais e teóricas (FPF e flambagem) permaneceram aproximadamente constantes, independentemente de seus comprimentos. Este tipo de falha,

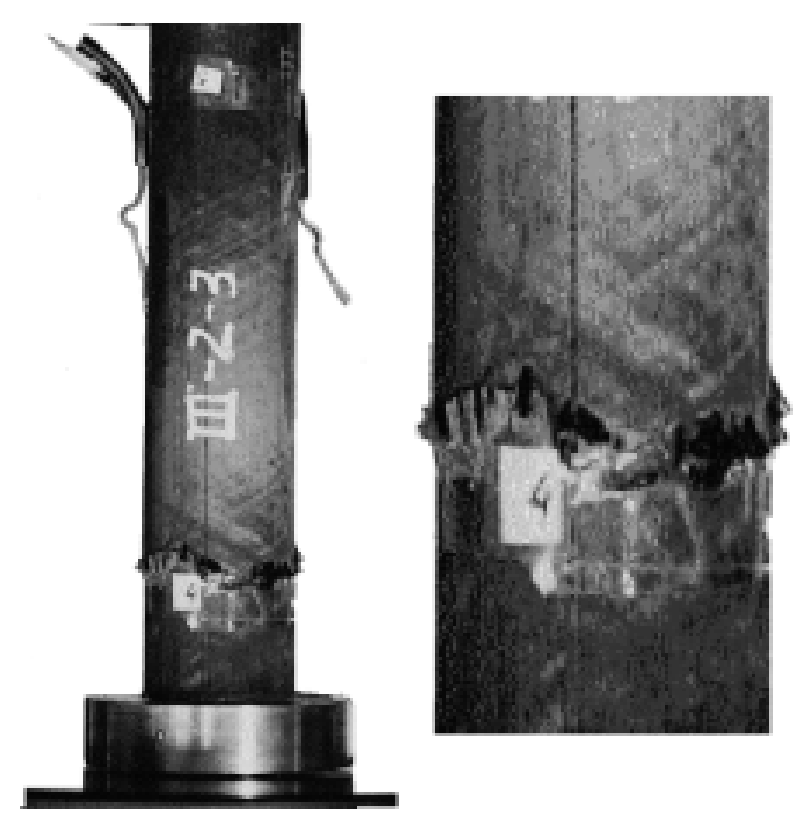

Figura 1. Ruptura de fibras perpendicularmente ao carregamento. 

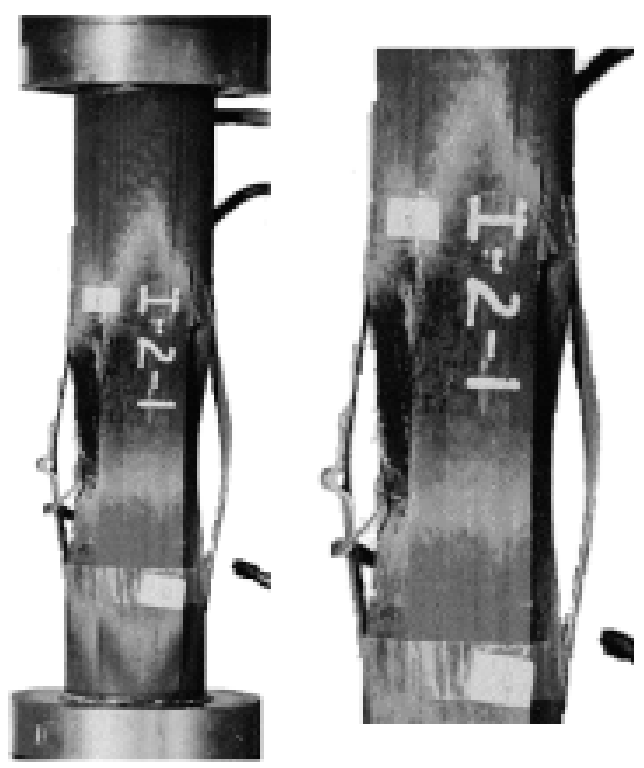

Figura 2. Flambagem local e fissuramento longitudinal da resina.

caracteriza-se por apresentar uma grande curvatura, fissuras da resina no eixo longitudinal e praticamente nenhuma quebra de filamentos (Figura 2).

Flambagem Elástica com Bifurcação: Este modo de falha ocorreu somente nos cilindros tipo IV (Figuras 3 e 4). Após aliviada a carga, os mesmos retornaram às suas formas iniciais, sem aparentemente apresentarem qualquer anomalia.

As características físicas e os resultados experimentais, para cada configuração de cilindro, encontram-se na Tabela 4, e os referentes às análises numéricas dos programas COMPSHELL, NASTRAN, estão indicados na Tabela 5. Os valores da rigidez longitudinal dos cilindros $\left(\boldsymbol{E}_{\boldsymbol{I C}}\right)$ foram obtidos com base nas leituras de dois extensômetros por corpo de prova. Estes valores, e os referentes às tensões de falha experimentais, listados na Tabela 4 , referem-se à média de cinco réplicas de corpos de prova para cada tipo de cilindro ${ }^{[9]}$.

Os resultados da Tabela 5 mostram que os valores das tensões de flambagem, obtidas pelo programa NASTRAN, em todos os casos, são mais elevadas que as tensões de falha encontradas nos ensaios experimentais. Fato este também verificado para os cilindros tipos I e IV, referentes aos cálculos de resistência (FPF, programa COMPSHELL) quando confrontados com as tensões de falha experimentais. Para os cilindros tipo II, as resistências teóricas, são menores que a média das tensões experimentais e para do tipo III são bem próximas das obtidas nos ensaios. Tanto o cálculo de resistência pelo critério de
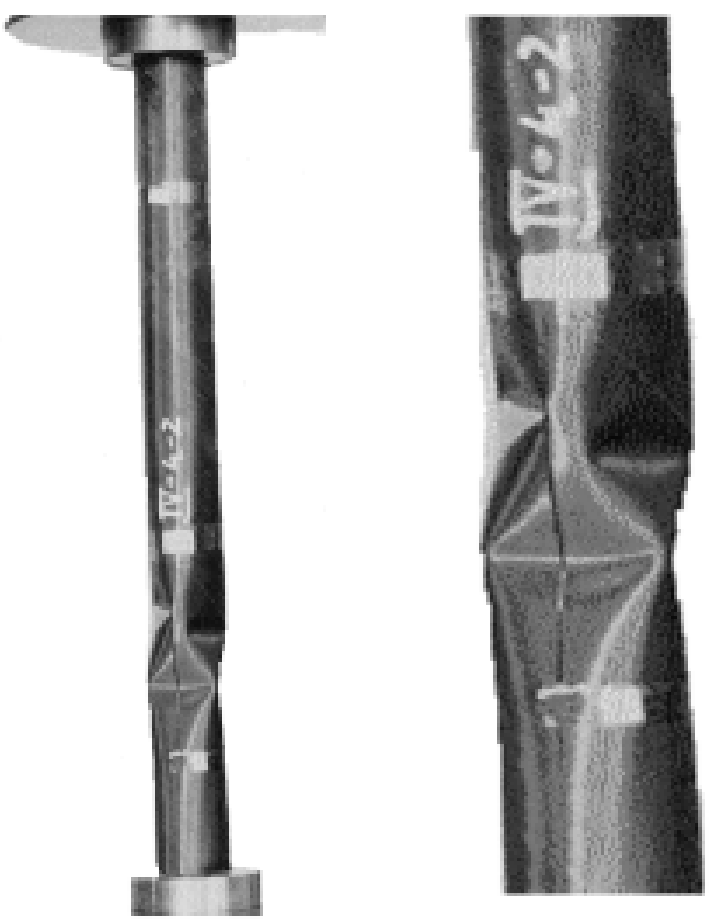

Figura 3. Flambagem elástica com bifurcação.
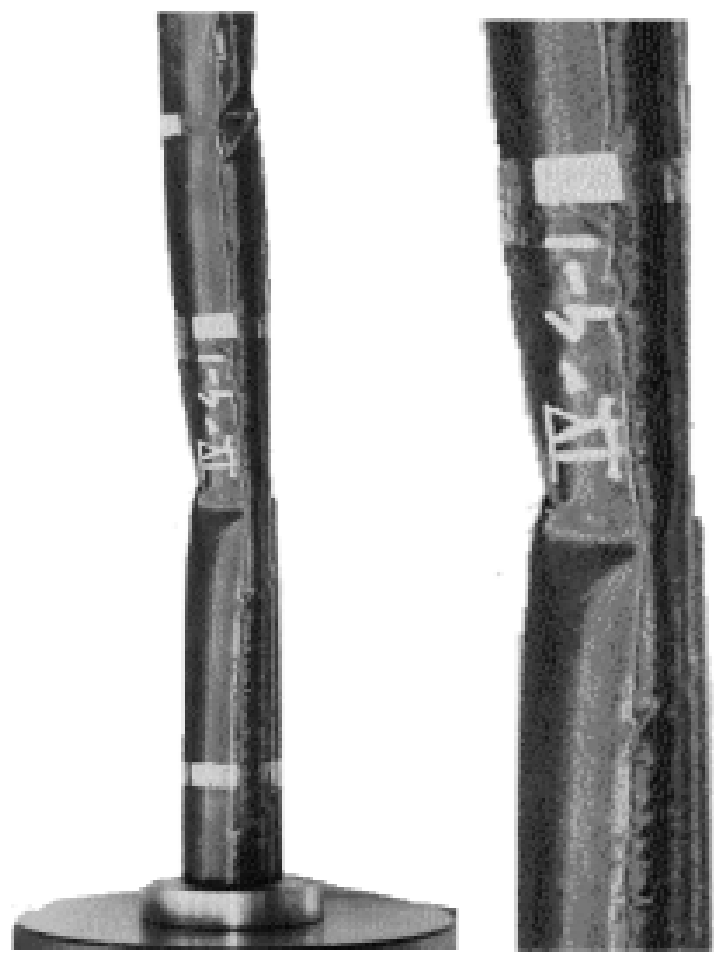

Figura 4. Flambagem elástica com bifurcação.

Hoffman ou pelo da Deformação Máxima, consideraram como falha total a falha da primeira camada ("first ply failure"). Sabe-se que o critério "first ply failure" (FPF) é conservativo, podendo subestimar a 
Tabela 4. Resultados dos ensaios.

\begin{tabular}{|c|c|c|c|c|}
\hline Identificação & $\begin{array}{c}\mathbf{L} \\
(\mathbf{m m})\end{array}$ & $\begin{array}{c}\text { Massa } \\
\text { (g) }\end{array}$ & $\begin{array}{c}\mathbf{E}_{1 \mathrm{C}} \\
(\mathrm{GPa}) \\
\text { Ensaio }\end{array}$ & $\begin{array}{c}\text { Tensão de Falha } \\
\text { Experimental } \\
\text { (MPa) }\end{array}$ \\
\hline $\mathrm{I}-1$ & 100 & 12,9 & 121 & $\begin{array}{c}217 \\
(18,3)^{(*)}\end{array}$ \\
\hline $\mathrm{I}-2$ & 200 & 25,3 & 121 & $\begin{array}{c}231 \\
(14,1)\end{array}$ \\
\hline $\mathrm{I}-3$ & 300 & 38,8 & 121 & $\begin{array}{c}206 \\
(20,3)\end{array}$ \\
\hline II-1 & 100 & 12,7 & 47 & $\begin{array}{c}227 \\
(24,2)\end{array}$ \\
\hline III-2 & 200 & 25,8 & 46 & $\begin{array}{c}214 \\
(21,9)\end{array}$ \\
\hline III-3 & 300 & 38,5 & 47 & $\begin{array}{c}210 \\
(23,8)\end{array}$ \\
\hline III-1 & 100 & 12,6 & 43 & $\begin{array}{c}245 \\
(27,4)\end{array}$ \\
\hline III-2 & 200 & 25,3 & 42 & $\begin{array}{c}232 \\
(29,1)\end{array}$ \\
\hline III-3 & 300 & 38,5 & 45 & $\begin{array}{c}198 \\
(26,4)\end{array}$ \\
\hline IV-4 & 450 & 19,2 & 16 & $\begin{array}{c}40 \\
(9,00)\end{array}$ \\
\hline
\end{tabular}

(*) Referem-se ao desvio padrão das tensões experimentais de falha.

tensão de fratura estática para determinados laminados ${ }^{[3]}$, fato este observado nos cilindros tipo II conforme os resultados da Tabela 5. Adicionalmente, dentre os dois critérios de falha empregados, nota-se na Tabela 5 que o da Deformação Máxima foi mais conservativo que o de Hoffman na grande maioria dos casos (9 dentre 10). Em se tratando de materiais tipicamente frágeis, como os laminados reforçados com fibras de carbono, sabe-se que o critério da Deformação Máxima conduz a resultados conser$\operatorname{vativos}^{[4,7]}$.

As análises numéricas utilizadas, consideram as estruturas perfeitas e sem desvios de fabricação, o que não representa as reais condições dos corpos de prova. Neste contexto, o fato de todas as tensões numéricas de flambagem superestimarem as tensões de falha experimentais, conforme os dados apresentados na Tabela 5 , é plenamente justificável ${ }^{[8]}$. Os valores das propriedades mecânicas dos materiais utilizados nos cálculos, foram obtidas a partir de corpos de prova planos, ensaiados segundo normas técnicas da ASTM, mas como as estruturas são cilíndricas, pode haver então alguma variação nos valores destas mesmas propriedades.

Os ensaios experimentais apresentados na Tabela 4 mostram que os valores das tensões de falha não variam demasiadamente com a mudança da orientação das camadas. Em cada lote de cinco corpos de prova ensaiados foi verificada uma dispersão nos resultados dentro de níveis aceitáveis (o máximo desvio padrão encontrado foi de $29,1 \mathrm{MPa}$, ou seja, $12,5 \%$ para o tipo III-2). O valor desta dispersão caracteriza a repetibilidade do processo de manufatura e indica que, para a obtenção de pequena variação nos resultados, os cilindros devem ser fabricados com critério e atenção.

Tabela 5. Resultados das análises numéricas, em relação às tensões experimentais de falha.

\begin{tabular}{ccccc}
\hline Identificação & Tensão de Falha Teórica & Tensão de & $\begin{array}{c}\text { Flambagem } \\
\text { (MPa) }\end{array}$ & $\begin{array}{c}\text { Tensão de Falha } \\
\text { Experimental } \\
\text { (MPa) }\end{array}$ \\
\hline I-1 & Hoffman & Deform. Max. & 568 & 217 \\
I-2 & 580 & 585 & 558 & 231 \\
I-3 & 580 & 523 & 556 & 206 \\
II-1 & 578 & 504 & 963 & 227 \\
II-2 & 144 & 131 & 757 & 214 \\
II-3 & 144 & 131 & 599 & 210 \\
III-1 & 144 & 131 & 970 & 245 \\
III-2 & 240 & 205 & 812 & 232 \\
III-3 & 240 & 206 & 676 & 198 \\
IV-4 & 240 & 206 & 162 & 40 \\
\hline
\end{tabular}




\section{Conclusões}

O objetivo principal deste trabalho foi o de caracterizar experimentalmente o comportamento de cilindros de carbono/epóxi, submetidos à cargas compressivas uniaxiais. Dentro desta proposta, foram ensaiados 47 cilindros de diferentes orientações de camadas e comprimentos e também realizada análise teórica para as configurações estabelecidas.

Tendo como base os resultados obtidos e os aspectos abordados neste trabalho, as seguintes observações devem ser consideradas:

- As imperfeições decorrentes do processo de fabricação influenciam diretamente o comportamento mecânico das estruturas. Assim, para se obter uma melhor correlação entre os resultados experimentais e numéricos, os cálculos teóricos devem levar em consideração a presença de imperfeições geométricas características do processo de fabricação utilizado. Esse cálculo seria extremamente complexo ${ }^{[8]}$ e requereria uma caracterização estatística das imperfeições presentes nos cilindros. Como alternativa, a análise experimental usada neste trabalho quantifica diretamente o efeito das imperfeições associadas ao processo de fabricação empregado, fornecendo dados de cargas limites essenciais ao projeto dessa classe de estrutura.

- A variação do comprimento dos cilindros para uma mesma configuração, mantendo-se fixos o raio e a espessura, não acarretou em variações significativas nos valores das tensões de falha e dos módulos de elasticidade em compressão;

- O Módulo de Elasticidade em Compressão $\left(E_{l C}\right.$, conforme Tabela 4$)$ tende a aumentar quando a orientação das camadas se alinha à direção de aplicação do carregamento, mas, de acordo com os resultados do modelo numérico implementado, torna a estrutura mais suscetível à flambagem.

- Para os cilindros que falharam por flambagem elástica com bifurcação (tipo IV), as Tensões de Flambagem Experimentais apresentaram valores bem abaixo das Tensões Teóricas. Nos demais casos, conforme indicam os resultados da Tabela 5, as correlações entre resultados experimentais e numéricos foram significativamente melhores.

\section{Agradecimentos}

Os autores agradecem ao Instituto de Aeronáutica e Espaço, IAE-CTA pela confecção e ensaio dos corpos de prova. $\mathrm{O}$ segundo autor agradece o apoio financeiro recebido do CNPq processo 300219/90-3.

\section{Referência Bibliográfica}

1. Tenek, L. T. \& Argyris, J. - "Finite Element Analysis for Composite Structures", Kluwer Academic Publishers, Boston (1998).

2. Bushnell, D. - "Computerized Analysis of Shells Governing Equations", Computers and Structures, Vol. 18, No. 3, p. 471 - 536, (1984).

3. Tsai, S. W. - "Composites Design", Think Composites, Dayton, (1990).

4. Levy Neto, F. - "The Behavior of ExternallyPressurised Composite Domes" Ph.D. Thesis, University of Liverpool, England (1991).

5. Davis, J. G., "Compressive Instability and Strength of Uniaxial Filament-reinforced Epoxy Tubes", NASA TN D-5697, NASA (1970).

6. Sun, G. \& Hansen, J. S. - Journal of Applied Mechanics, 55, p. 136 (1988).

7. Mistry, J. - Composite Structures, 20, p. 83 (1992).

8. Brush, D. O. \& Almroth, B. O. - "Buckling of Bars, Plates and Shells", McGraw Hill, New York, 1975.

9. Gonçalves, A. - “Análise Experimental da Estabilidade e Resistência de Cilindros de Carbono/Epóxi Submetidos a Cargas Compressivas Uniaxiais" Tese de Mestrado, Instituto Tecnológico de Aeronáutica, Brasil (1997).

10. Vizzini,A. J., Lagace, P. A. - "The Role of Ply Buckling in the Compressive Failure of Graphite/Epoxy Tubes" in: Proceedings of the $25^{\text {th }}$ Structures, Structural Dynamics and Materials Conference, Paper 84-0963, Palm Springs (1984).

11. Almeida, S. F. M., and Hansen, J. S., Journal of Applied Mechanics, Vol. 64, No. 4, (1997), pp. 772780 .

Recebido: 20/03/01

Aprovado: 08/08/01 